\title{
Adverse Effect of Different Exercise Types on Ovarian Reserve
}

\author{
Guner Cicek $^{1}$, Umit Gorkem $^{2}$, Faruk Yamaner ${ }^{1}$, Abdullah Gullu $^{1}$, Esin Gullu ${ }^{1}$ \\ ${ }^{1}$ Hitit University, Faculty of Sports Science, Turkey \\ ${ }^{2}$ Hitit University, Faculty of Medicine, Department of Obstetrics and Gynecology, Turkey \\ Correspondence: Guner Cicek, Hitit University, Faculty of Sports Science, ,Turkey. E-mail: gunercicek@ hitit.edu.tr
}

Received: November 26, 2018

doi:10.11114/jets.v7i1.3894

\author{
Accepted: December 18, 2018 \\ Online Published: December 21, 2018
}

URL: https://doi.org/10.11114/jets.v7i1.3894

\begin{abstract}
In this study, it was aimed to determine the negative effect of different types of exercise on ovarian reserve of sedentary women. A total of 40 sedentary women who met the criteria for admission to work were categorized as aerobic exercise (AE;

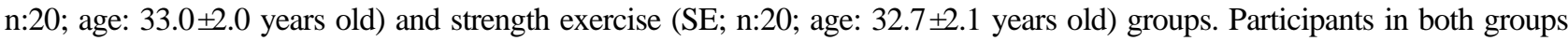
received two different exercises of 60-minute with an intensity of 60-70\% for 16 weeks and 4 days per week. Participants' anthropometric (body weight, BMI, waist and hip circumference, waist and hip rate (WHR)), estradiol (E2), luteinizing hormone (LH) follicle stimulating hormone (FSH), and levels antimülerian hormone (AMH), which used as over-reserve indicators, were measured for before and after exercise. Comparisons of these parameters were evaluated at $\mathrm{p}<0.01$ and 0.05 levels. The mean of anthropometric and biochemical parameters of the AE and SE groups were statistically similar in before exercise. After exercise program, body weight, BMI, waist and hip circumference values of both groups were significantly decreased $(\mathrm{p}<0.01$ and $p<0.05)$. There was no significant correlation between AMH, E2, FSH and LH levels of the AE group $(p>0.05)$. In the SE group, there was a decrease in AHM level $(p<0.05)$ and an increase in FSH level $(\mathrm{p}<0.01)$. There was no significant correlation between $\mathrm{E} 2$, and LH levels of the SE group ( $p>0.05)$. As a results; strength exercise triggers to decrease in serum AMH levels and to increase in serum FSH levels. For this reason, strength exercise practices can have negative consequences for fertility, especially for women with lower ovarian reserve values.
\end{abstract}

Keywords: exercise, sedentary woman, ovarian reserve, anti-müllerian hormone

\section{Introduction}

The ovarian reserve is indicated by the number and quality of the ovarian follicle pool and declines with the age increase. Hence, resulting in the decrease of a woman's fertility function (Te Velde et al., 1998; Di Mario et., 2018). In women, fertility begins to decline after the age of 30 (Scheffer et al., 2018), but there are could be individual change in the age of menopause and in the age of below fertility (Te Velde, et al., 1998), therefore chronological age is not a good predictor of ovarian reserve (Di Mario et., 2018). Some of biological markers have been identifying to estimation the ovarian reserve such as the evaluation of serum levels of follicle stimulating hormone (FSH), Anti-Mullerian hormone $(\mathrm{AMH})$, inhibin B, estradiol, (E2) and antral follicle count (AFC). These are the commonly used ovarian reserve markers for assessing ovarian reserve in the women of reproductive age (Broer et al., 2009; Broekmans et al., 2006; Nelson et al., 2009; Kiranmayee et al., 2017).

Recently, AMH has been shown to help predict early ovarian follicle loss and the onset of menopause; and is defined as a reliable predictor of ovarian reserve (Victoria et al., 2018). In addition to, AMH is increasingly used in clinical practice for both prognostic purposes, (Broer et al., 2010; Pigny et al., 2006; Dolleman et al., 2013) such as prediction of ovarian response during in vitro fertilization treatment (IVF) and diagnostic purposes such as the identification of women with premature ovarian failure (POF) or polycystic ovarian syndrome (PCOS) can be possible (Broer et al., 2009; Knauff et al., 2009). With regular exercise, weight loss has been shown to improve the metabolic function and hormonal profiles. The exercises also usually lead to a significant increase in fertility (Norman et al., 2004; Al-Eisa et al., 2017). It has been assumed that there is significant evidence that physical activity improves the quality of life in the general population. However, there is insufficient evidence for the effect of physical activity and quality of life on improving fertility in infertile women (Anderson, et al., 2010; Saremi, et al., 2013; Orio et al., 2013).

In this study, it was aimed to determine the negative effect of different types of exercise on ovarian reserve of sedentary women. 


\section{Material and Method}

\section{Participants}

A total of 40 healthy sedentary women participated in this study. The participants were randomly categorized into two groups; aerobic exercise $(\mathrm{AE} ; \mathrm{n}=20)$ and strength exercise $(\mathrm{SE} ; \mathrm{n}=20)$. Exclusion criteria were $>35$ years of age, pregnancy, diagnosed cases of premature ovarian failure, current use of medications influencing reproductive functions, hyperprolactinemia, conditions known to affect reproductive functions including oophorectomy and / or hysterectomy, endometriosis, pelvic surgery, smoking, alcohol, ovarian masses, use of hormonal contraceptives, systemic diseases including heart diseases, thyroid diseases, diabetes mellitus, inflammatory and autoimmune diseases. This study approved from The Clinical Research Ethics Committee in Medical Faculty of Ondokuz Mayis University (215/435) in accordance with the policy statement of the Turkey Ministry of Health.

\section{Exercise intervention}

Participants in both groups admitted two different exercises of 60-minute with an intensity of 60-70\% for 16 weeks and 4 days per week. Heart rate was monitored continuously during all sessions, with the goal of maintaining an intensity of 60-70\% max HR. Exercises severity was calculated separately for each participant. The exercise program for two groups was undertaken 4 days of each week during 16 consecutive weeks and every exercise session was of $1 \mathrm{~h}$ duration. Aerobic exercises program consisted of warm-up exercises (10min), main exercises including (30-40 min, basic movements step-aerobic) and cold-down $(10 \mathrm{~min})$. Strength exercises program; warm-up exercises (10 min), main exercises including (30-40 min strength exercises provide basically more strength for lower and upper extremities include (1-8 weeks; 3 sets/day, 10 repetitions/ set, 1-2min resting interval), (8-16 weeks; 4 sets/day, 10 repetitions/ set, $1-2 \mathrm{~min}$ resting interval and end of the exercises cold-down (10 $\mathrm{min})$.

\section{Anthropometric measurements}

Anthropometric measurements were taken two times as at baseline, after 16 weeks and at the end of the study. Height was measured to the nearest $0.1 \mathrm{~cm}$ on a stadiometer, Body weight of participants was measured by the weighing instrument in kilograms $\pm 0.01 \mathrm{~kg}$ and body mass index (BMI)was calculated by dividing weight $(\mathrm{kg}$ ) by height squared $\left(\mathrm{m}^{2}\right)$. Hip circumference, waist circumference were measured and waist-hip rate (WHR) was calculated.

\section{Blood sampling and analysis}

In this study were taken two times blood samples as baseline and after 16 weeks' exercise. The blood samples of the participants were taken from antecubital vein after an overnight fasting between 8:00 AM and 10:00 AM in the early follicular phase on days 2 to 5 . The samples allowed to clot completely at room temperature, after that centrifuged within $30 \mathrm{~min}$ at $1500 \mathrm{~g}$ for $4 \mathrm{~min}$. Serums were analyzed on a daily basis for follicle stimulating hormone (FSH), luteinizing hormone (LH) and estradiol (E2) by the electrochemiluminescence immunoassay (ECLIA) method using an autoanalyzer (Cobas 6000, E 601 Roche Diagnostics, GmbH, Mannheim, Germany). The serums for AMH measurements were frozen at $-20{ }^{\circ} \mathrm{C}$ within 2 hours for 5 days at mostly and then analyzed. All analyses of AMH samples were also completed on a weekly basis by ECLIA technique using an autoanalyser (Cobas 6000, E 601 Roche Diagnostics, GmbH, Mannheim, Germany). All of the analyses were implement at the Duzen Laboratory in Ankara, Turkey

\section{Statistical analyses}

All analyses were performed using SPSS software version 20 packet program. Data are expressed as the arithmetic means and standard deviation (SD). The distributions of all of the continuous variables for normality were tested using Shapiro-Wilk tests. The data were normally distributed. The evaluation within the group was made by Paired Samples-t test. Statistical significance were accepted as $\mathrm{p}<0.01$ and $\mathrm{p}<0.05$.

\section{Results}

Table 1. Characteristics of the study group (mean $\pm \mathrm{SD})$

\begin{tabular}{lcc}
\hline & Aerobic exercise group $(\mathbf{n}=\mathbf{2 0})$ & Strength exercise group $(\mathbf{n}=\mathbf{2 0})$ \\
\hline Age $($ years $)$ & $33.0 \pm 2.0$ & $32.7 \pm 2.1$ \\
Height $(\mathbf{m})$ & $1.62 \pm 4.51$ & $1.64 \pm 3.12$ \\
Weight $(\mathbf{c m})$ & $67.10 \pm 7.61$ & $68.50 \pm 4.19$ \\
BMI $\left(\mathbf{k g} / \mathbf{m}^{2}\right)$ & $25.36 \pm 2.51$ & $25.65 \pm 2.17$ \\
\hline
\end{tabular}


Table 2. Anthropometric measurements of participants baseline and after 16 weeks

\begin{tabular}{lcccc}
\hline & \multicolumn{2}{c}{ Aerobic exercise group $(\mathbf{n}=20)$} & \multicolumn{2}{c}{ Strength exercise group $(\mathbf{n}=20)$} \\
\hline Variables & Baseline & 16 weeks & baseline & 16 weeks \\
Weight $(\mathbf{c m})$ & $67.10 \pm 7.61$ & $63.75 \pm 8.45^{*}$ & $68.50 \pm 4.19$ & $64.35 \pm 5.85^{* *}$ \\
BMI $(\mathbf{k g} / \mathbf{m} 2)$ & $25.36 \pm 2.51$ & $24.02 \pm 2.84^{*}$ & $25.65 \pm 2.17$ & $24.42 \pm 1.83^{* *}$ \\
Waist $(\mathbf{c m})$ & $85.30 \pm 6.87$ & $82.10 \pm 7.32^{* *}$ & $84.30 \pm 6.94$ & $81.25 \pm 7.51^{* *}$ \\
Hip $(\mathbf{c m})$ & $109.55 \pm 5.64$ & $104.95 \pm 5.06^{* *}$ & $109.90 \pm 5.32$ & $105.00 \pm 5.67^{* *}$ \\
WHR $(\mathbf{c m})$ & $0.78 \pm 0.67$ & $0.78 \pm 0.77$ & $0.76 \pm 0.56$ & $0.77 \pm 0.83^{* *}$ \\
\hline
\end{tabular}

The mean difference is significant level: $* * P<0.01 * P<0.05$

Abbreviation: BMI: body mass index, WHR: waist/hip ratio,

In table 2, when we compare the pre- and post-exercise values in both groups, there is statistically significant decreases were found in body weight, BMI, waist and hip values $(\mathrm{p}<0.01$ and $\mathrm{p}<0.05)$.

Table 3. Both of groups ovarian reserve parameters baseline and after 16 weeks exercises.

\begin{tabular}{lcccc}
\hline & \multicolumn{2}{c}{ Aerobic exercise group $(\mathbf{n}=\mathbf{2 0})$} & \multicolumn{2}{c}{ Strength exercise group $(\mathbf{n}=\mathbf{2 0})$} \\
\hline Variables & Baseline & 16 weeks & baseline & 16 weeks \\
E2 $(\mathbf{p g} / \mathbf{m L})$ & $40.15 \pm 29.19$ & $37.05 \pm 26.26$ & $41.18 \pm 17.44$ & $44.15 \pm 19.62$ \\
LH (IU/L) & $7.09 \pm 3.46$ & $8.85 \pm 8.62$ & $6.93 \pm 4.35$ & $7.91 \pm 3.70$ \\
FSH (IU/L) & $5.32 \pm 1.92$ & $4.98 \pm 1.78$ & $4.71 \pm 1.62$ & $6.07 \pm 2.11^{* *}$ \\
AMH (ng/dL) & $2.14 \pm 3.22$ & $2.19 \pm 2.46$ & $4.16 \pm 3.08$ & $3.48 \pm 2.32^{*}$ \\
\hline
\end{tabular}

The mean difference is significant level: $* * P<0.01 * P<0.05$

Abbreviation: E2: Estradiol, LH: Luteining hormone, FSH: Follicle stimulating hormone,

AMH: Antimullerian hormone

Table 3 shows that there was a small reduction in the levels of E2 and FSH after the workouts in the aerobic exercise group; however, the differences between the pre- and post-exercise levels were not statistically significant. Similarly, in the same group, the differences between the levels of LH and AMH were not statistically significant, either ( $p>0.05$ ). On the other hand, in the strength training group, there was a statistically significant increase in FSH levels $(\mathrm{p}<0.01)$ and a significant decrease in the AMH levels $(\mathrm{p}<0.05)$. The difference in the E2 and LH levels were not statistically significant in the strength training exercise group $(p>0.05)$.

\section{Discussion}

This study was conducted to examine the positive and negative effects of aerobic and strength training exercises, performed four times a week during 16 weeks, on the ovarian reserve parameters in sedentary women.

Our study has found that the body weight, BMI, and waist and hip circumferences reduced statistically in both groups of exercised women. Previous studies in the literature have shown that both aerobic and strength training exercises resulted in a reduction in the body weight, BMI, and waist and hip circumferences positively (Maiya, et al., 2008; Martins et al., 2010; Morencos et al., 2012; Atuegbu, et al., 2017).

Previous studies have suggested a potential role of weight loss as the main factor in restoring fertility, although to a minimal extent (Palomba et al., 2007; Ramlau-Hansen et al., 2007; Nouri, et al., 2016). Similarly, the significant reduction in the waist circumference after 3 and 12 weeks of aerobic workouts suggests the potential role of visceral fat reduction in facilitating ovulation. The changes in the amount of visceral fat, occurring in response to lifestyle changes, result in reduced androgen levels and an alleviated insulin resistance regardless of the body weight (Wright et al., 2004; Nouri, et al., 2016; Al-Eisa et al., 2017). One study found that exercise led to a reduction in the BMI levels, and in the total and android fat mass, as well as, decreasing the heightened insulin sensitivity in women (Moran, et al., 2011). Our study found that the body weight, BMI, and waist circumference were reduced favorably after the exercising period of 16 weeks in our study, suggesting that these parameters did not affect the ovarian reserve parameters. Despite the significant reduction in BMI, AMH serum levels were negatively affected in the strength training group. BMI has been previously reported to be negatively correlated with serum AMH levels potentially in women of reproductive age (Freeman et al., 2007; Su, et al., 2008).

The examination of hormone parameters of ovarian reserve in our study showed that the post-exercise E2 and FSH levels were reduced to a smaller extent compared to those quantified before within aerobic exercise group. However, the differences were not statistically significant different. Similarly, in the same group, the differences between the levels of LH and AMH were not statistically significant, either ( $p>0.05$ ). In the strength training group; the FSH levels were increased from $4.71 \pm 1.62$ to $6.07 \pm 2.11$ whereas, the $\mathrm{AMH}$ levels decreased from $4.16 \pm 3.08$ to $3.48 \pm 2.32$ with a statistically significant difference. 
A study reported that an intense workout of at least 30 minutes per day reduced the risk of infertility associated with ovulation in women (Mohan, 2005). However, extreme levels of exercise may affect the reproductive functions in a negative way, such as inducing primary or secondary amenorrhea by involving the hypothalamic-pituitary-gonadal axis and suppressing the luteinizing hormone (LH) and the follicle stimulating hormone (FSH) (Winterer et al., 1984; Atuegbu, et al., 2017). Kiranmayee, et al. (2017) conducted a study on 162 women at the ages between 19-42 and found that a moderate degree of physical activity was associated with higher levels of AMH in women under 30 years. Another study reported that intense exercising for an hour in a week was associated with a lower risk of infertility related to ovulation by $7 \%$ in normal-weight women (Rich-Edwards et al., 2002). While there are many studies describing the positive effects of physical activity on ovarian reserve markers and fertility outcomes in women of reproductive age (Kucuk et al., 2010; Saremi et al., 2013; Anderson et al., 2010), there is another study reporting that an intense level of physical activity is associated with adverse effects on fertility outcomes (Zanker, 2006). Al-Eisa et al. (2017) have a study on obese patients and reported that a 12-week moderate-degree aerobic exercise led to estrogen storage, reduced the body fat, and induced steroid hormone synthesis, which in turn increases FSH and decreases the level of AMH.The results of our study share similarities with the study of Al-Eisa et al., (2017). The 16-week strength exercises caused a weight reduction, increased the levels of FSH, and reduced AMH levels in sedentary women.

\section{Conclusion}

E2, LH, FSH, and AMH levels of hormone markers of the ovarian reserve did not significantly change in sedentary women, who performed long-term aerobic exercises. The strength exercises, on the other hand, induced a reduction in AMH levels and an increase in serum FSH levels in women. These findings suggest that strength exercises may affect fertility unfavorably, especially in women with low ovarian reserves

\section{Acknowledgement}

This research was supported by The Commission of Scientific Research Projects of Hitit University. Project number is BYO19002.15.002

\section{References}

Al-Eisa, E., Gabr, S. A., \& Alghadir, A. H. (2017). Effects of supervised aerobic training on the levels of anti-Mullerian hormone and adiposity measures in women with normo-ovulatory and polycystic ovary syndrome. J. Pak. Med. Assoc, 67, 499.

Anderson, K., Nisenblat, V., \& Norman, R. (2010). Lifestyle factors in people seeking infertility treatment-a review. Australian and New Zealand journal of obstetrics and gynaecology, 50(1), 8-20. https://doi.org/10.1111/j.1479-828X.2009.01119.x

Atuegbu, C. M., Meludu, S. C., Dioka, C. E., Onyenekwe, C. C., Onuegbu, J. A., Onah, C. E., \& Udo, J. N. (2017). Effect of moderate-vigorous intensity physical exercise on female sex hormones in premenopausal university students in Nnewi, Nigeria. International Journal of Research in Medical Sciences, 2(4), 1516-1520. https://doi.org/10.5455/2320-6012.ijrms20141151

Broekmans, F. J., Kwee, J., Hendriks, D. J., Mol, B. W., \& Lambalk, C. B.(2006). A systematic review of tests predicting ovarian reserve and IVF outcome. Hum Reprod Update. 12, 685-718. https://doi.org/10.1093/humupd/dml034

Broer, S. L., Mol, B. W., Hendriks, D., \& Broekmans, F. J. (2009). The role of antimullerian hormone in prediction of outcome after IVF: Comparison with the antral follicle count. Fertil Steril. 91, 705-714. https://doi.org/10.1016/j.fertnstert.2007.12.013

Broer, S. L., Mol, B., Dolleman, M., Fauser, B. C., \& Broekmans, F. J. (2010). The role of anti-Müllerian hormone assessment in assisted reproductive technology outcome. Current Opinion in Obstetrics and Gynecology, 22(3), 193-201. https://doi.org/10.1097/GCO.0b013e3283384911

Di Mario, C., Petricca, L., Gigante, M. R., Barini, A., Barini, A., Varriano, V., \& Gremese, E. (2018). Anti-Müllerian hormone serum levels in systemic lupus erythematosus patients: Influence of the disease severity and therapy on the ovarian reserve. Endocrine, 1-7.

Dolleman, M., Verschuren, W. M. M., Eijkemans, M. J. C., Dollé, M. E. T., Jansen, E. H. J. M., Broekmans, F. J. M., \& Van der Schouw, Y. T. (2013). Reproductive and lifestyle determinants of anti-Müllerian hormone in a large population-based study. The Journal of Clinical Endocrinology \& Metabolism, 98(5), 2106-2115. https://doi.org/10.1210/jc.2012-3995

Freeman, E. W., Gracia, C. R., Sammel, M. D., Lin, H., Lim, L. C. L., \& Strauss III, J. F. (2007). Association of anti-mullerian hormone levels with obesity in late reproductive-age women. Fertility and sterility, 87(1), 101-106. 
https://doi.org/10.1016/j.fertnstert.2006.05.074

Kiranmayee, D., Praveena, T., Himabindu, Y., Sriharibabu, M., Kavya, K., \& Mahalakshmi, M. (2017). The Effect of Moderate Physical Activity on Ovarian Reserve Markers in Reproductive Age Women Below and Above 30 Years. J Hum Reprod Sci. 2017 Jan-Mar;10(1), 44-48.

Knauff, E. A., Eijkemans, M. J., Lambalk, C. B., ten Kate-Booij, M. J., Hoek, A., Beerendonk, C. C., \& de Jong, F. H. (2009). Anti-Mullerian hormone, inhibin B, and antral follicle count in young women with ovarian failure. The Journal of Clinical Endocrinology \& Metabolism, 94(3), 786-792. https://doi.org/10.1210/jc.2008-1818

Kucuk, M., Doymaz, F., \& Urman, B. (2010). Effect of energy expenditure and physical activity on the outcomes of assisted reproduction treatment. Reproductive biomedicine online, 20(2), 274-279.

https://doi.org/10.1016/j.rbmo.2009.11.011

Maiya, A. G., Sheela, R. K., \& Kumar, P. (2008). Exercise-induced weight reduction and fertility outcomes in women with polycystic ovarian syndrome who are obese and infertile: A preliminary report'. Journal of exercise science and physiotherapy, 4(1), 30.

Martins, R. A., Veríssimo, M. T., e Silva, M. J. C., Cumming, S. P., \& Teixeira, A. M. (2010). Effects of aerobic and strength-based training on metabolic health indicators in older adults. Lipids in Health and Disease, 9(1), 76. https://doi.org/10.1186/1476-511X-9-76

Mohan, B. S. (2005). Mid-luteal phase plasma progesterone levels in spontaneous and clomiphene citrate induced conception cycles. J Obstet Gynecol India, 55, 4

Moran, L. J., Harrison, C. L., Hutchison, S. K., Stepto, N. K., Strauss, B. J., \& Teede, H. J. (2011). Exercise Decreases Anti-Müllerian Hormone in Anovulatory Overweight Women with Polycystic Ovary Syndrome-A Pilot Study. Hormone and metabolic research, 43(13), 977-979. https://doi.org/10.1055/s-0031-1291208

Morencos, E., Romero, B., Peinado, A. B., González-Gross, M., Fernández Godoy, C., Gómez Candela, C., \& Benito, P. J. (2012). Effects of dietary restriction combined with different exercise programs or physical activity recommendations on blood lipids in overweight adults. Nutrición hospitalaria. 27(6), 1916-1927

Nelson, S. M., Yates, R. W., Lyall, H., Jamieson, M., Traynor, I., Gaudoin, M., \& Fleming, R. (2009). Anti-Müllerian hormone-based approach to controlled ovarian stimulation for assisted conception. Human reproduction, 24(4), 867-875. https://doi.org/10.1093/humrep/den480

Norman, R. J., Noakes, M., Wu, R., Davies, M. J., Moran, L., \& Wang, J. X. (2004). Improving reproductive performance in overweight/obese women with effective weight management. Human reproduction update, 10(3), 267-280. https://doi.org/10.1093/humupd/dmh018

Nouri, M., Aghadavod, E., Khani, S., Jamilian, M., Amiri Siavashani, M., Ahmadi, S., \& Asemi, Z. (2016). Association between BMI and gene expression of anti-Müllerian hormone and androgen receptor in human granulosa cells in women with and without polycystic ovary syndrome. Clinical endocrinology, 85(4), 590-595. https://doi.org/10.1111/cen.13098

Orio, F., Muscogiuri, G., Ascione, A., Marciano, F., Volpe, A., La, G. S., \& Palomba, S. (2013). Effects of physical exercise on the female reproductive system. Minerva endocrinologica, 38(3), 305-319.

Palomba, S., Giallauria, F., Falbo, A., Russo, T., Oppedisano, R., Tolino, A., \& Orio, F. (2007). Structured exercise training programme versus hypocaloric hyperproteic diet in obese polycystic ovary syndrome patients with anovulatory infertility: a 24-week pilot study. Human Reproduction, 23(3), 642-650. https://doi.org/10.1093/humrep/dem391

Pigny, P., Jonard, S., Robert, Y., \& Dewailly, D. (2006). Serum anti-Mullerian hormone as a surrogate for antral follicle count for definition of the polycystic ovary syndrome. The Journal of Clinical Endocrinology \& Metabolism, 91(3), 941-945. https://doi.org/10.1210/jc.2005-2076

Ramlau-Hansen, C. H., Thulstrup, A. M., Nohr, E. A., Bonde, J. P., Sørensen, T. I. A., \& Olsen, J. (2007). Subfecundity in overweight and obese couples. Human reproduction, 22(6), 1634-1637. https://doi.org/10.1093/humrep/dem035

Rich-Edwards, J. W., Spiegelman, D., Garland, M., Hertzmark, E., Hunter, D. J., Colditz, G. A., \& Manson, J. E. (2002). Physical activity, body mass index, and ovulatory disorder infertility. Epidemiology, 184-190. https://doi.org/10.1097/00001648-200203000-00013

Saremi, A., Shavandi, N., \& Dezfulian, M. (2013). The effect of physical activity on serum levels of anti-mullerian hormone and fertility parameters in reproductive age women. 
Scheffer, J. A. B., Scheffer, B., Scheffer, R., Florencio, F., Grynberg, M., \& Lozano, D. M. (2018). Are age and anti-Müllerian hormone good predictors of ovarian reserve and response in women undergoing IVF?. JBRA assisted reproduction, 22(3), 215-220. https://doi.org/10.5935/1518-0557.20180043

Su, H. I., Sammel, M. D., Freeman, E. W., Lin, H., DeBlasis, T., \& Gracia, C. R. (2008). Body size affects measures of ovarian reserve in late reproductive age women. Menopause (New York, NY), $15(5), 857$. https://doi.org/10.1097/gme.0b013e318165981e

Te Velde, E. R., Dorland, M., \& Broekmans, F. J. (1998). Age at menopause as a marker of reproductive ageing. Maturitas, 30(2), 119-125. https://doi.org/10.1016/S0378-5122(98)00067-X

Te Velde, E. R., Scheffer, G. J., Dorland, M., Broekmans, F. J., \& Fauser, B. C. J. M. (1998). Developmental and endocrine aspects of normal ovarian aging. Molecular and cellular endocrinology, 145(1-2), 67-73. https://doi.org/10.1016/S0303-7207(98)00171-3

Victoria, M., Labrosse, J., Krief, F., Cédrin-Durnerin, I., Comtet, M., \& Grynberg, M. (2018). Anti Müllerian Hormone: more than a biomarker of female reproductive function. Journal of Gynecology Obstetrics and Human Reproduction. https://doi.org/10.1016/j.jogoh.2018.10.015

Winterer, J., Cutler Jr, G. B., \& Loriaux, D. L. (1984). Caloric balance, brain to body ratio, and the timing of menarche. Medical hypotheses, 15(1), 87-91. https://doi.org/10.1016/0306-9877(84)90011-2

Wright, C. E., Zborowski, J. V., Talbott, E. O., McHugh-Pemu, K., \& Youk, A. (2004). Dietary intake, physical activity, and obesity in women with polycystic ovary syndrome. International Journal of Obesity, $28(8), 1026$. https://doi.org/10.1038/sj.ijo.0802661

Zanker, C. L. (2006). Regulation of reproductive function in athletic women: an investigation of the roles of energy availability and body composition. British journal of sports medicine, 40(6), 489-490. https://doi.org/10.1136/bjsm.2004.016758

\section{Copyrights}

Copyright for this article is retained by the author(s), with first publication rights granted to the journal.

This is an open-access article distributed under the terms and conditions of the Creative Commons Attribution license which permits unrestricted use, distribution, and reproduction in any medium, provided the original work is properly cited. 